\title{
Carbon Fibre Composites: Integrated Electrochemical Sensors for Wound Management
}

\author{
Duncan Sharp", Stephen Forsythe and James Davis \\ ${ }^{1}$ School of Science and Technology, Nottingham Trent University, \\ Nottingham, NG11 8NS, UK
}

Manuscript No JB-07-10-0352

Submitted to:

The Journal of Biochemistry; Biotechnology: Biosensors and Bioelectrics

Regular Paper

http://jb.oxfordjournals.org/

* To whom correspondence should be addressed. T: +44 (0) 115848 3218; E: duncan.sharp@ntu.ac.uk 


\section{Abstract}

The applicability of employing a carbon fibre mesh as an electrochemical sensing substructure for assessing urate transformations within wound exudates is evaluated. Prototype sensor assemblies have been designed and their response characteristics towards uric acid and other common physiological components are detailed. Modification of the carbon fibre sensor through surface anodisation and the application of cellulose acetate permselective barriers have been shown to lead to optimized responses and much greater sensitivity ( $1440 \%$ increase) and specificity. These could enable the accurate periodic monitoring of uric acid in wound fluid. The performance characteristics of the composite sensors in whole blood, serum and blister fluid have been investigated.

\section{Keywords}

Carbon fibre, smart bandage, urate, uric acid, wound infection 


\section{Introduction}

The threat of wound infection is an ever present hazard in modern healthcare whether the patient is being treated locally or within a hospital environment. A recent survey has put the incidence of hospital acquired infections (HAI's) relating to surgical wound management at around $10 \%[1,2]$ and it has been estimated that complications arising from these can increase the length of hospitalisation to between 6-13 days $[3,4]$. There are obvious implications for the patient and health service with the cost to the UK NHS alone within the billion pound region [3]. In many cases, the origin is simply the colonisation of the wound by adventitious opportunistic bacteria such as Pseudomonas aeruginosa or Staphylococcus aureus [5-7] as a consequence of poor hygiene. Irrespective of the cause, there is a need for a more intelligent approach to wound management. While this clearly dictates that improvements are made to basic hygiene, technology also has a part to play and recent advances in nano-particle science have seen the development of antibacterial dressings, frequently Silver based [8]. Nevertheless, the inherent adaptability of micro-organisms means that there remains a need for a failsafe system that can alert either the patient or the health care professional to the advent of a potential infection. The present communication has sought to explore the use of an electrochemical sensing system capable of measuring urate directly within the wound fluid as an indirect marker for assessing both the physiological response to the injury and, importantly, as a generic indicator for the presence of bacterial colonisation. Whilst surgical wounds as a whole may be considered; of particular importance regarding infection and other complications is the management of thermal burn injuries. These are ascribed to the large, open surface of such wounds, especially following debridment, and the generation of reactive oxygen species (ROS's) and inflammatory mediators which may induce systemic injury [9]. 
The underlying rationale behind the choice of urate lies in the fact that the molecule has a significant physiological role for the patient [10] and is degraded by certain pathogenic and opportunist pathogenic bacteria including the aforementioned infectants [11].

It could be anticipated that its inherent anti-oxidant properties may mean that the local concentration and fluctuations therein reflect the nature of ongoing oxidative stress processes within the body, which may lead to serious and potentially-fatal systemic complications e.g. Cardiac mitochondrial damage [12]. Thus, uric acid determination could provide a semi quantitative diagnostic assessment of the severity of the local and systemic injury; or the effectiveness of the antioxidant treatment as part of the requisite fluid resuscitation.

Another possibility is that the local consumption of urate could, in principle, occur where certain bacterial colonies effectively metabolise the urate. The critical point in the latter rests upon the fact that urate is the final end product of purine catabolism within humans and hence the relatively large concentration within serum $(150-420 \mu \mathrm{M}$ [13]). Many organisms, both pathogens and opportunist pathogens, have the ability to metabolise uric acid; importantly, the two major causes of burn wound infection: Staphylococcus aureus and Pseudomonas aeruginosa are included [11,14-16]. Both of which have been reported to rapidly metabolise uric acid, via microbial uricase synthesis. Microbial uricase catabolises uric acid to allantoin [17], as is commonplace in the human gastro-intestinal tract.

Therefore, it could be envisaged that reductions in urate concentration may provide a generic indicator for monitoring the change from bacterial contamination to colonization. A substantial depletion of urate may consequently provide a vital, early warning flag that can alert the patient or healthcare professional such that remedial action can be taken prior to the onset of "critical colonization" and the subsequent wound infection [18]. As well as a decrease in mortality, it is reasonable to assume that this may help reduce the generation of antibiotic resistant bacterial strains, by the theory, the fewer bacteria treated with an antibiotic, the fewer mutant organisms expressed. 
The foundations of the sensor design rest upon the exploitation of a carbon fibre mesh both as the detection element and the transduction conduit. The electro-oxidation of urate at various forms of carbon is well documented and there are numerous analytical methodologies incorporating such [19-24]. The uses of carbon fibre electrodes for biomedical applications are also well documented for the detection of: nitric oxide [25], lactate dehydrogenase [26], perphenazine [27], haemoglobin [28], ascorbate, catechol and indole [29], chloramphenicol [30] and uric acid $[31,32]$ However the aforementioned detection of uric acid was performed in-vitro, using diluted sample and a large surface area sensor. Single fibre electrodes are commonly used for in-vivo monitoring for many other analytes, commonly used to measure analytes within the brain; acetylcholine and choline [33], dopamine [34-36], acetaminophen [37], nitric oxide [38] and glucose [39]. The challenge, and the emphasis of the current investigation, is to develop a processing strategy that allows the material to be harnessed in a way that can allow the rapid production of sensors that could be easily incorporated within a smart bandage and which can facilitate reagentless and periodic monitoring of urate directly within a range of biofluids.

The core rationale is outlined in Figure 1A where the carbon fibre sheets are thermally sandwiched between polyester laminate. The underlying carbon is selectively exposed to function as the sensing element by laser etching through the encapsulating sheet - which can then be divided up into individual sensing structures and thereby offers an opportunity for mass manufacture. The proposed application of the laminate as a smart sensor is shown in Figure 1B. The system is integrated within a conventional adhesive plaster - replacing the absorbent pad component traditionally associated with the latter.

The principal aim of this investigation was to determine the potential applicability of the process outlined in Figure 1 through assessing the response of the carbon fibre composite sensor to urate in various biofluids of direct relevance to wound management. 


\section{Experimental Details}

Materials: All reagents were of the highest grade available and used without further purification. Stock solutions of uric acid (typically $10 \mathrm{mM}$ ) were prepared in $0.1 \mathrm{M} \mathrm{NaOH}$. All other solutions were prepared using Britton-Robinson buffer (acetic, boric and phosphoric acids - each at a concentration of $0.04 \mathrm{M}$ ) adjusted to $\mathrm{pH} 7$ through the addition of sodium hydroxide. Standard solutions were generally prepared in deionised water from an Elgastat (Elga, UK) water system and refrigerated when not in use. Toray carbon fibre cloth was purchased from E-Tek Inc (USA) and used as received. Lamination pouches (Rexel UK) were a commercial stationary variety with a film thickness of $75 \mu \mathrm{m}$ each side. Copper Shielding tape (100 $\mu \mathrm{m}$ thick, adhesive backed) was obtained from RS electronics.

Instrumentation: Electrochemical measurements were conducted using a $\mu$ Autolab type III computer controlled potentiostat (Eco-Chemie, Utrecht, The Netherlands) using a two electrode configuration consisting of the carbon fibre assembly working electrode, a chloridised silver wire as the combined counter/reference electrode.

Sensor Construction: Laminated carbon fibre prototypes were prepared by thermally sandwiching carbon fibre sheet between sleeves of a pre-etched (1 or $2 \mathrm{~mm}$ diameter window) resin-polyester lamination pouch using a commercially available laminator. Electrical connection to the carbon film was made through the presence of a strip of copper shielding tape. The electrodes were baked at $100^{\circ} \mathrm{C}$ for 1 hour in order to ensure the complete permeation of the resin between the fibres within the laminate. This is necessary to ensure the mechanical integrity and coherence of the seal between the sensing fibre layer and the insulating polyester sheath such that no solvent creep or de-lamination would occur during extended monitoring periods times (up to 18 replicate scans over 30 minutes or 7 replicate scans over 120 minutes) 


\section{Results and Discussion}

The morphology of the laser patterned laminate - carbon composite was examined using scanning electron microscopy with the interface between the exposed fibre substructure and the insulating laminate detailed in Figure 2. The sensing element is effectively a random assembly of discrete and amalgamated fibres presenting a large 3-dimensional network and is in marked contrast to the planar designs found in conventional macro or micro sized urate sensor formats [40,41]. The initial analytical characterisation of the applicability of the network towards the sensing of urate was conducted in buffered solution - containing up to $500 \mu \mathrm{M}$ ascorbate. The addition of latter is significant in that it is ubiquitous within biofluids and easily electro-oxidised at potentials not dissimilar to those required for urate detection. Square wave voltammograms detailing the response of the carbon network to equimolar urate and ascorbate are detailed in Figure 3 (dashed line). A single, broad peak is observed with no resolution between the two compounds.

Pre-treatment of the carbon fibre sensor through oxidation in $0.1 \mathrm{M}$ sodium hydroxide $(+2 \mathrm{~V}, 10 \mathrm{~min}$. $)$ yielded a very different response. A single sharp peak is observed at $+0.23 \mathrm{~V}$ which is attributed solely to the oxidation of urate. It has been previously shown that the anodic fracturing of carbon substrate as a consequence of such pre-treatment gives superior resolution between ascorbate and urate and markedly reduces the electron transfer kinetics of the former such that, under normal physiological concentrations, it provides a negligible contribution to the voltammetric profile. The anodizing of the carbon fibre created a substantial gain $(1440 \%$ increase) in the magnitude of the urate peak as detailed in Figure 3. Confirmation that the sharp peak at $+0.23 \mathrm{~V}$ is indeed urate with no contribution from ascorbate was provided by repeating the experiment but with a markedly increased concentration of ascorbate. Square wave voltammograms detailing the response to $100 \mu \mathrm{M}$ urate in the presence of ascorbate $(2.2 \mathrm{mM})$ are 
shown in Figure 3 solid line. The ascorbate emerges as a broad peak $(+0.03 \mathrm{~V})$ to the left of the sharp urate process. This highlights the fact that even in the presence of massive ascorbate concentration - it is still possible to obtain an unambiguous assessment of urate concentration and is in marked contrast to the result obtained with the un-modified carbon fibre (Figure 3 - dashed line). The anodisation effect leads to the exfoliation of the fibre structure of the mesh increasing the surface area at the nanoscale and increasing the population of hydrophilic (typically hydroxyl and carboxylic acid) functionalities on the resulting exposed surface and confirmed by XPS studies. The delamination effect also has the influence of creating more edge plane sites which also serves to increase the electron transfer rate and hence improve the response to urate $[42,43]$.

The influence of real biofluids on the sensor response was again assessed using square wave voltammetry; the real biofluids were used early in this prototype development, as these are fluids in which a smart-bandage sensor is required to function. The responses of the untreated and modified fibre sensors assemblies to whole blood are detailed in Figure 4A. In this instance, the untreated blood was applied directly to the sensing surface and the measurement conducted almost immediately. The response of the un-modified fibre sensor shows effectively no discrimination between the different physiological components with a single broad peak found at $+0.69 \mathrm{~V}$. The pre-anodised sensor, however, displays a peak profile similar to that observed in the control buffer solution (Figure $\mathbf{3}$ - solid line). The magnitude of the peaks could be enhanced through increasing the degree of surface pre-treatment prior to applying the blood. Thus, extending the pre-anodisation time to 30 minutes results in a markedly enhanced signal (Figure 4A has been baseline offset for clarity) with three, clearly resolved peak processes. The first ($0.12 \mathrm{~V})$ is attributed to the redox groups within the fibre substructure, the second $(+0.23 \mathrm{~V})$ is the urate and the third $(+0.66 \mathrm{~V})$ is liable to be a combination of other, less easily oxidised biological components such as tyrosine, tryptophan as well as other purines. This was corroborated by comparing the response to urate in the presence of tryptophan (Figure 4B). The emergence of a 
second peak at $+0.53 \mathrm{~V}$ is located in a similar position to that observed with the whole blood sample. Similar responses were observed with tyrosine with near identical peak positions between tyrosine and tryptophan highlighting both the limitation of the sensor for speciation in such complex fluids but, in the present instance, the supreme advantage of facilitating the almost unique discrimination of urate from the other blood constituents.

Given that the sensor can clearly detect urate in a complex biofluid, the next issue to be addressed relates to whether or not it is indeed capable of monitoring urate beyond the initial scan. The intended application requires periodic scanning of the biofluid for differences in urate concentration and hence alert the patient / clinical staff to the possibility of wound colonisation. Serum samples were used in this instance to avoid the complications of clotting and the need for exogenous agents to prevent such (e.g. Lithium heparin). This would allow multiple replicate measurements on the same sensor assembly over a prolonged period and hence would mimic the conditions under which a prototype could be expected to operate. A square wave voltammogram detailing the initial response to the application of the serum sample is shown in Figure $5 \mathbf{A}$. The urate peak is again clearly resolved and is consistent with both the control urate solution and the responses observed in whole blood. The variation in peak height as a function of replicate scans (upto 18 replicates: same sensor, same sample) is highlighted in Figure 5B (solid circles). It can be seen that the peak height response decreases markedly with increasing measurements. It was envisaged that the sustained decay in the response could be attributed to the fouling of the electrode surface by the extra-cellular components - principally protein and fats - effectively reducing the active sensing area and hence the response.

To counter this problem, the electrode surface was coated with cellulose acetate to act as a protective permselective barrier acting by size exclusion (prepared by drop casting from an ethanol solution). The response characteristics of this second modification have been included within Figure 5B (white circles) for comparison. There is an initial decay in response, similar to that observed with the uncoated anodised fibre mesh, but, in contrast to the latter, the response 
soon stabilises. It is possible that the initial responses are simply a consequence of the equilibration of the anodised fibre in the new medium. The difference in response characteristics (normalised to the peak height measured on the first scan) between the cellulose acetate modified sensor and the uncoated, anodised, system is marked with only a minor loss in performance ( $\sim 20 \%$ ) observed with the former whereas the latter suffers significantly ( $>60 \%$ decrease) over 18 replicate scans. The application of this semi-permeable coating reduced the signal current by between $3 \mathrm{x}$ and $5 \mathrm{x}$ due to decreasing surface area, as to be expected. The subsequent results however show this does not have a substantial impact on the overall sensitivity of the sensor detection as sufficient currents have been detected, even for uric acid concentrations far below the physiological range for biofluids. Whilst carbon fibre and cellulose acetate have previously been used in conjunction; this is the first reported use of them together for detection of uric acid or for use in wound management, again highlighting this novel approach to smart bandage development.

The last hurdle in the preliminary assessment of the applicability of the sensing system was to determine whether or not it could detect urate in a blister wound - typical of the open wound liable to be subject to common bacterial infection. A square wave voltammogram detailing the response of the anodised sensor system towards the blister exudate is shown in Figure 5C. The profile is again similar to that found with the other biofluids and highlights the potential for applying the sensor system within a number of biomedical contexts where it may be necessary to monitor wound status.

To ensure that accurate quantification of uric acid is possible a series of standard curves were run throughout the investigation, whilst the lower and more physiologically relevant range $(0-500 \mu \mathrm{M})$ fit a linear equation $(y=0.025 x+0.561)$, with an $R$-squared value of 0.97 , it was found possible to extend the analytical range upto $1 \mathrm{mM}$ but fitting a more complex, one-site saturation standard curve (Figure 6A). $(\mathrm{y}=(\operatorname{Bmax} . \mathrm{x}) /(\mathrm{Kd}+\mathrm{x})$ where $\mathrm{Bmax}=1.5654$ and $\mathrm{Kd}=178.4328)$ with R-squared 0.99. The linear increase in uric acid concentration (Figure 6B), as measured by the 
proposed electrochemical system, correlates with the spiking of the biofluid (serum) highlighting the ability of the sensor to monitor minor fluctuations of the uric acid concentration. The three serum spikes $(25,50$ and $100 \mu \mathrm{M}$ urate) were chosen to show both the sensitivity to urate concentration changes and by doubling the spike each time allow a simple spiked-serum standard plot to be established. The relative change of the uric acid is highlighted as this is of most importance for the proposed application.

\section{Conclusions}

The carbon fibre system has shown to be capable of easy integration into a robust and versatile sensing system. Modification of the carbon network either pre or post lamination provides superior resolution and sensitivity of urate detection across a number of biomedical contexts. Periodic monitoring has been shown to be feasible and hence the system could facilitate short to moderate term wound management in a smart bandage application for the monitoring of bacterial metabolism of uric acid. Hence, in addition to the in-vitro quantification of uric acid in common biofluids this direct electrochemical sensor has proven successful for the detection of uric acid in blister/wound fluid, a previously unexplored application, and the closest to a truly in-vivo uric acid carbon fibre sensor. The only thorough investigation using carbon fibre electrodes to measure uric acid in biofluids (serum) [32] was performed using much larger electrode diameters (Typically $5 \mathrm{~mm}-25 \mathrm{x}$ greater surface area!) and using diluted serum samples for the analysis, however, the proposed design offers similar specificity, and due to having a much smaller surface area, substantially less uric acid would be metabolised by the electrochemical oxidation (via detection) thereby cause minimal affects on wound physiology. 
Given the reagent-less and stable nature of the sensors proposed within, there are no issues with stability or storage conditions unlike enzyme based-sensors and throughout this investigation the batch produced sensors had shown no indications of instability or degradation. Longer term studies of shelf life would however be required but are beyond the scope of the present preliminary - proof of concept investigations presented herein. The ease with which the sensor can be fabricated, the unambiguous and sensitive nature of the signal is clearly an advantage over conventional urate measurements systems. Therefore the proposed electrochemical system clearly proffers a strong foundation for quantitative smart bandage technologies.

\section{Acknowledgements}

The authors thank the EPSRC for supporting this work. 


\section{References}

1. Wilson, J. (2001) Infection Control in Clinical Practice, Elsevier Science, London.

2. Surveillance of Surgical Site Infection in English Hospitals: a national surveillance and quality improvement programme. Health Protection Agency-NINSS, 2002.

3. Plowman, R. (2000) The socioeconomic burden of hospital acquired infection. Euro Surveill 5, 49-50

4. Fisher, J. (1994) The plague Makers. Simon and Schuster, New York.

5. Mousa H.A.-L. (1997) Aerobic, anaerobic and fungal burn wound infections. Journal of Hospital Infection 37, 317-323

6. Chai, J., Sheng, Z., Yang, H., Diao, L. and Li, L. (2000) Successful treatment of invasive burn wound infection with sepsis in patients with major burns. Chinese Medical Journal 113, 11421146

7. Vidhani, S., Mehndiratta, P.L. and Mathur, M.D. (2001) Study of methicillin Resistant S. aureus (MRSA) isolates from high riskpatients. Indian Journal of Medical Microbiology 19(2)

8. Atiyeh, B.S., Costagiola, M., Hayek, S.N. and Dibo, S. A. (2007) Effect of silver on burn wound infection control and healing: Review of literature. Burns 33, 139-148

9. Ono, O., Gunji, H., Zhang, J.-Z., Maruyama, K. and Kaneko, F. (1995) A study of cytokines in burn blister fluid related to wound healing. Burns 21, 352-355

10. Becker, B.F. (1993) Towards the physiological function of uric acid. Free Radical Biology and Medicine 14, 615-631

11. Rouf, M. A. and Lomprey, R.F. (1968) Degradation of Uric Acid by Certain Aerobic Bacteria. Journal of Bacteriology, Sept, 617-622

12. Zang, Q., Maass, D. L., White, J. and Horton, J.W. (2007) Cardiac mitochondrial damage and loss of ROS defense after burn injury: the beneficial effects of antioxidant therapy. $J$ Appl Physiol 102, $103-112$

13. Diagnostic Services Handbook (2005) Nottingham City Hospital NHS trust, UK

14. Smith, R.F.(1970) Nitrogen Requirements and Uricolytic Activity of Cutaneous Bacteria. Applied Microbiology 19, 643-648

15. Bongaerts, G.P.A., Sin, I.L., Peters, A.L.J. and Vogels, G.D. (1977) Purine degradation in Pseudomonas aeruginosa and Pseudomonas testerone. Biochimica et Biophysica Acta 499, 111118

16. Abdel-Fattah, Y.R., Saeed, H.M., Gohar, Y.M. and El-Baz, M.A. (2005) Improved production of Pseudomonas aeruginosa uricase by optimization of process parameters through statistical experimental design. Process Biochemistry 40, 1705-1714

17. Adámek, V., Králová, B., Suchová, M., Valentova, O. and Demnerova, K. (1989) Purification of microbial uricase. Journal of Chromatography 497, 268-275

18. Ayton, M. (1985) Wound care: wounds that wont heal. Nursing Times 81, 16-19 
19. Cai, X., Kalcher, K. and Neuhold, C. (1994) Simultaneous Determination of Uric-Acid, Xanthine and Hypoxanthine with an Electrochemically Pretreated Carbon-Paste Electrode. Fresenius Journal of Analytical Chemistry 348, 660-665

20. Shi, K. and Shiu, K.K. (2001) Determination of uric acid at electrochemically activated glassy carbon electrode. Electroanalysis 13, 1319-1325

21. Ye, J.S., Wen, Y. and De Zhang, W. (2003) Selective voltammetric detection of uric acid in the presence of ascorbic acid at well-aligned carbon nanotube electrode. Electroanalysis 15, 16931698

22. Lu, G.H., Jiang, L.Y. and Song, F. (2005) Determination of uric acid and norepinephrine by chitosan-multiwall carbon nanotube modified electrode. Electroanalysis 17, 901-905

23. Yan, Q.P., Zhao, F.Q. and Li, G.Z. (2006) Voltammetric determination of uric acid with a glassy carbon electrode coated by paste of multiwalled carbon nanotubes and ionic liquid.

Electroanalysis 18 , 1075-1080

24. Safavi, A., Maleki, N. and Moradlou, O. (2006) Simultaneous determination of dopamine, ascorbic acid, and uric acid using carbon ionic liquid electrode. Analytical Biochemistry 359 , 224229

25. Katrlic, J. and Zaleskova, P. (2002) Nitric oxide determination by amperometric carbon fibre microelectrode. Bioelectrochemistry 56, 73-76

26. Ju, H.X., Dong, L. and Chen, H.Y. (1996) Amperometric determination of lactate dehydrogenase based on a carbon fibre microcylinder electrode modified covalently with Toluidine Blue $\mathrm{O}$ by acylation. Talanta $\mathbf{4 3}, 1177-1183$

27. Liu. D. and Jin, W. (2003) Amperometric detection of perphenazine at a carbon fibre microbundle electrode by capillary zone electrophoresis. Journal of Chromatography B 789, 411-415

28. Ju, H., Sun, H. and Chen, H. (1996) Properties of poly-B-aminoanthraquinone modified carbon fibre electrode as a basis for haemoglobin biosensor. Analytica Chimica Acta 327, 125-132

29. Crespi, F. (1996) Carbon Fibre micro-electrode and in vitro or in brain slices voltammetric measurement of ascorbate, catechol and indole oxidation signals: influence of temperature and physiological media. Biosensors and Bioelectronics 11, 743-749

30. Agui, L., Guzman, A., Yanez-Sedeno, P. and Pingarron, J.M. (2002) Voltammetric determination of chloramphenicol in milk at electrochemically activated carbon fibre microelectrodes. Analytica Chimica Acta 461, 65-73

31. Dutt, J.S.N., Cardosi, M.F., Wilkins, S., Livingstone, C. and Davis, J. (2006) Characterisation of carbon fibre composites for decentralised biomedical testing. Materials Chemistry and Physics $\mathbf{9 7}$, 267-272

32. Dutt, J.S.N., Livingstone, C., Cardosi, M.F., Wilkins, S.J. and Davis, J. (2006) A clinical assessment of direct electrochemical urate measurements. Talanta 68, 1463-1468 
33. Schuvailo, O.N., Dzyadevych, S.V., El'skaya, A.V., Gautier-Sauvigne, S., Csoregi, E., Cespuglio, R. and Soldatkin, A.P. (2005) Carbon fibre-based microbiosensors for in vivo measurements of acetylcholine and choline. Biosensors and Bioelectronics 21, 87-94

34. Suard-Chagny, M.-F. (2004) In vivo monitoring of dopamine overflow in the central nervous system by amperometric techniques combined with carbon fibre electrodes. Methods 33, 322-329

35. Dressman, S.F., Peters, J.L. and Michael, A.C. (2002) Carbon fibre microelectrodes with multiple sensing elements for in vivo voltammetry. Journal of Neuroscience Methods 119, 75-81

36. Yavich, L. and Tiihonen, J. (2000) In vivo voltammetry with removable carbon fibre electrodes in freely-moving mice: dopamine release during intracranial self-stimulation. Journal of Neuroscience Methods 104, 55-63

37. Logman, M.J., Budygim, E.A., Gainetdinov, R.R. and Wightman, R.M. (2000) Quantification of in vivo measurements with carbon fibre microelectrodes. Journal of Neuroscience Methods $\mathbf{9 5}$, 95-102

38. Park, J.-K., Tran, P.H., Chao, J.K.T., Ghodadra, R., Rangarajan, R. and Thakor, N.V. (1998) In vivo nitric oxide sensor using non-conducting polymer-modified carbon fibre. Biosensors and Bioelectronics 13, 1187-1195

39. Netchiporouk, L.I., Shram, N.F., Jaffrezic-Renault, N., Martelet, C. and Cespuglio, R (1996) In vivo Brain Glucose Measuremtns: Differential normal pulse voltammetry with enzyme-modified carbon fibre microelectrodes. Anal. Chem. 68 4358-4364

40. Zen, J.-M., Jou, J.-J. and Hangovan, G. (1998) Selective volatammetric method for uric acid detection using pre-anodized Nafion-coated glassy carbon electrodes. Analyst 123, 1345-1350

41. Chen, J.-C., Chung, H.-H., Hsu, C.-T., Tsai, D.-M., Kumar, A.S. and Zen, J.-M. (2005) A disposable single-use electrochemical sensor for the detection of uric acid in human whole blood. Sensors and Actuators $B$ 110, $364-369$

42. Ramesha, G.K.; Sampath S., (2007) Exfoliated graphite oxide modified electrode for the selective determination of picomolar lead, Electroanalysis 19, 2472-2478

43. Ishifune, M., Suzuki, R., Mima, Y., Uchida, K., Yamashita,N., Kashimura, S. (2005), Electrochimica Acta $\mathbf{5 1}$ 14-22 


\section{Figure Legends}

Figure 1. A) Schematic of the lamination process and B) the prototype sensing assembly. (1) wound aperture, (2) adhesive plaster backing, (3) insulating laminate, (4) carbon fibre sensor, (5) sensing window, (6) cellulose acetate barrier, (7) combined $\mathrm{Ag} / \mathrm{AgCl}$ reference/counter electrode, $(8)$ contacts to measuring device.

Figure 2. Scanning electron micrograph of the carbon fibre mesh / laminate composite.

Figure 3. Square wave voltammogram comparing the response of an untreated carbon fibre in equimolar $100 \mu \mathrm{M}$ UA and AA, with an anodized electrode in $100 \mu \mathrm{M}$ UA and 2.2 mM AA

Figure 4. A) Square wave voltammograms detailing the response of the untreated carbon fibre (dashed line) and the anodized fibre (after 15 and 30 min pre-treatment) towards whole blood. B) Response of $400 \mu \mathrm{M}$ urate in the presence and absence of $100 \mu \mathrm{M}$ Tryptophan.

Figure 5. A) Square wave voltammograms detailing the response of an anodized carbon fibre sensing assembly towards human serum. B) Influence of cellulose acetate on the periodic response monitoring of urate in serum. C) Response of the pre-treated, cellulose acetate coated, sensor towards blister fluid.

Figure 6. A) Calibration plot, with data, for the quantification of uric acid (in $\mathrm{pH} 7$ buffer) using an anodized carbon fibre sensor. B) Uric acid measurements in a serially spiked (25, 50 and $100 \mu \mathrm{M}$ urate) serum sample 

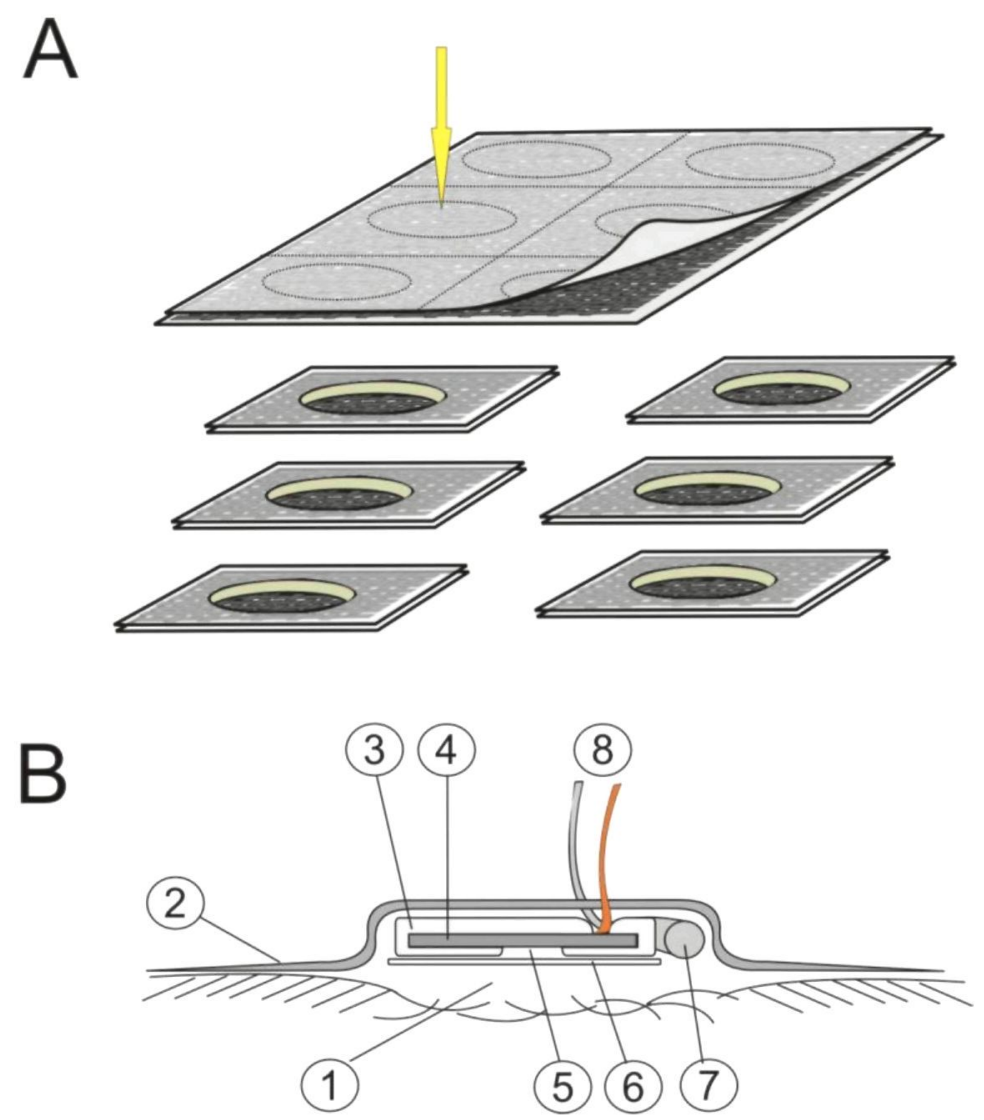

Figure 1. A) Schematic of the lamination process and B) the prototype sensing assembly. (1) wound aperture, (2) adhesive plaster backing, (3) insulating laminate, (4) carbon fibre sensor, (5) sensing window, (6) cellulose acetate barrier, (7) combined $\mathrm{Ag} / \mathrm{AgCl}$ reference/counter electrode, (8) contacts to measuring device. 


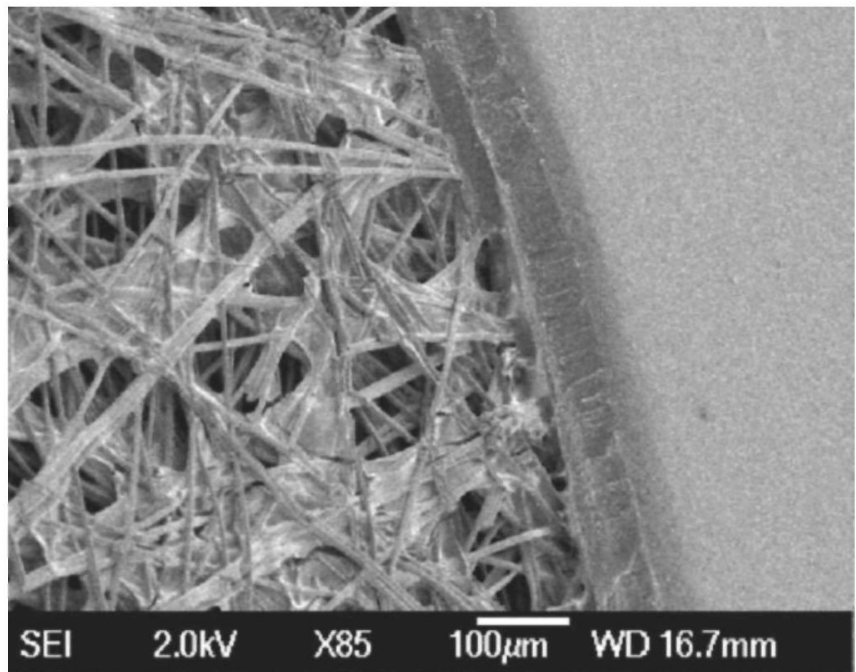

Figure 2. Scanning electron micrograph of the carbon fibre mesh / laminate composite. 


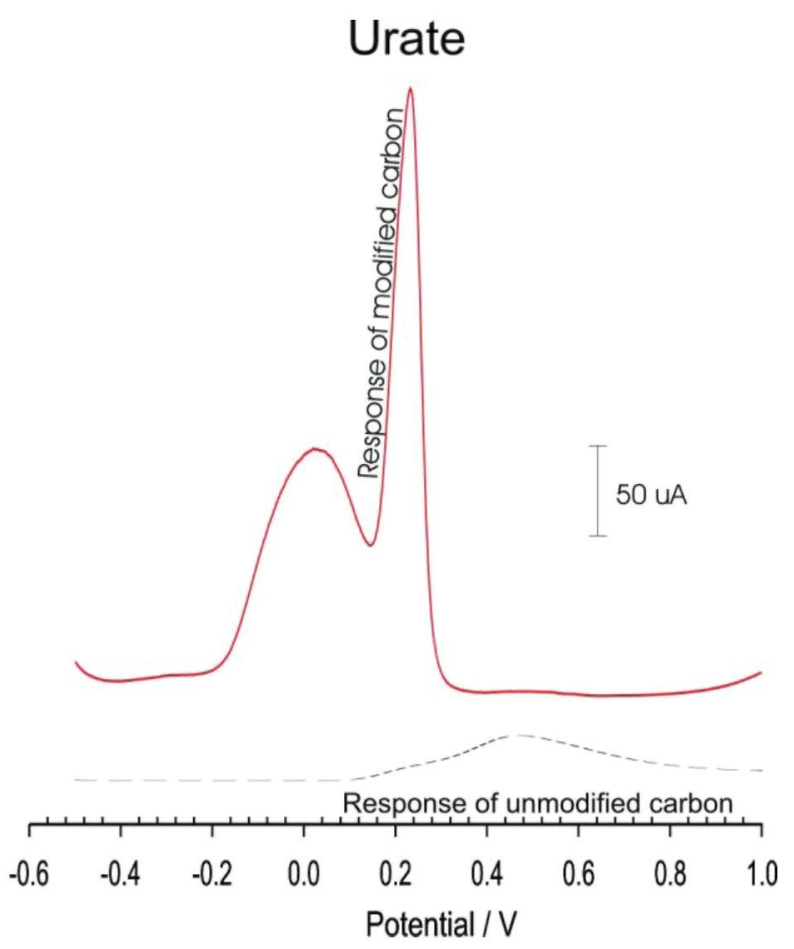

Figure 3. Square wave voltammogram comparing the response of an untreated carbon fibre in equimolar $100 \mu \mathrm{M}$ UA and AA, with an anodized electrode in $100 \mu \mathrm{M} \mathrm{UA}$ and $2.2 \mathrm{mM} \mathrm{AA} \mathrm{(pH}$ 7.0) 


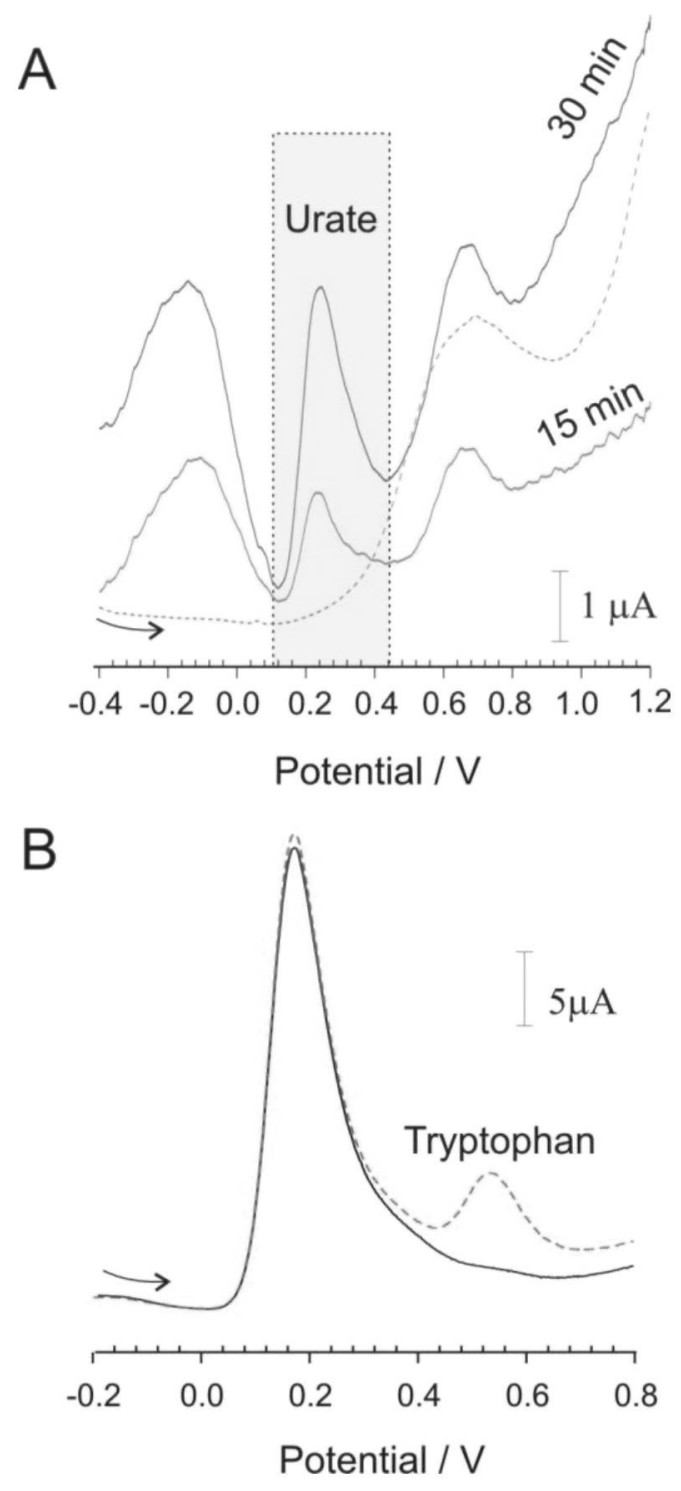

Figure 4. A) Square wave voltammograms detailing the response of the untreated carbon fibre (dashed line) and the anodized fibre (after 15 and 30 mins pre-treatment) towards whole blood. B) Response of $400 \mu \mathrm{M}$ urate in the presence and absence of $100 \mu \mathrm{M}$ tryptophan. 

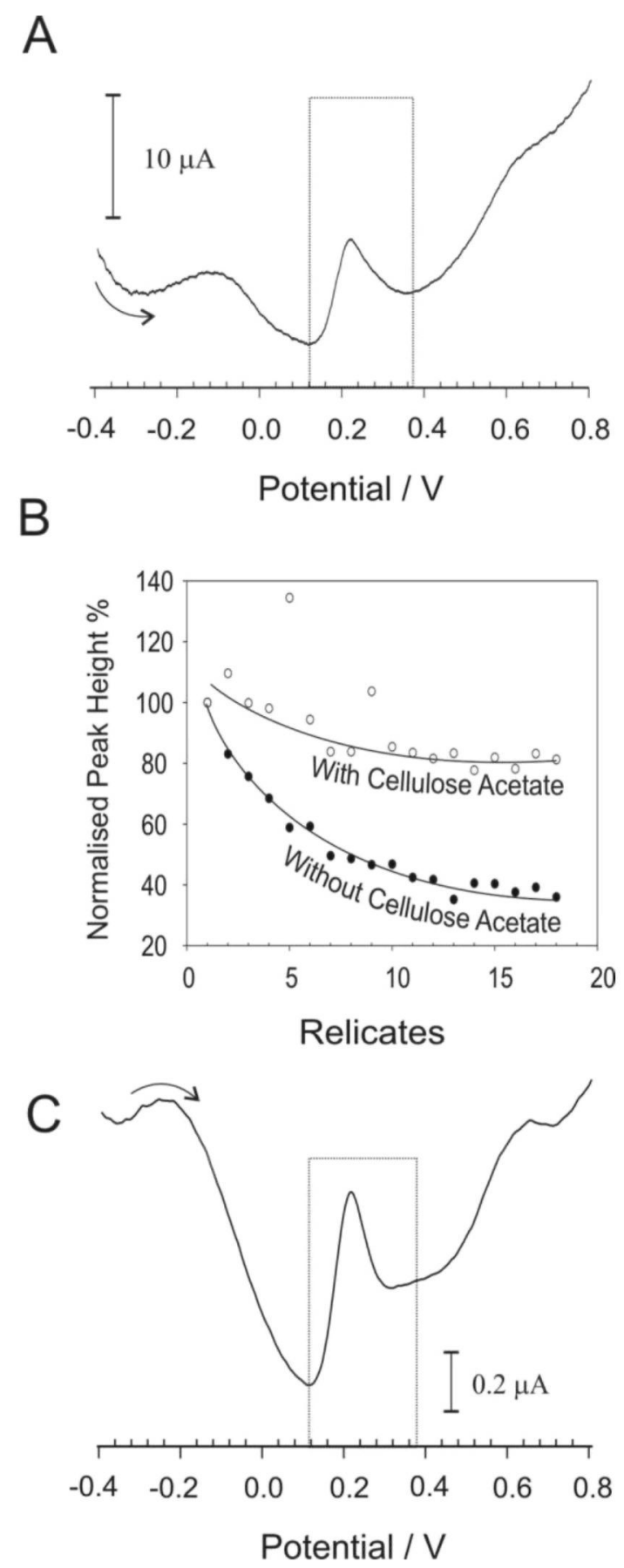

Figure 5. A) Square wave voltammograms detailing the response of an anodized carbon fibre sensing assembly towards human serum. B) Influence of cellulose acetate on the periodic response monitoring of urate in serum. C) Response of the pre-treated, cellulose acetate coated, sensor towards blister fluid. 
A)

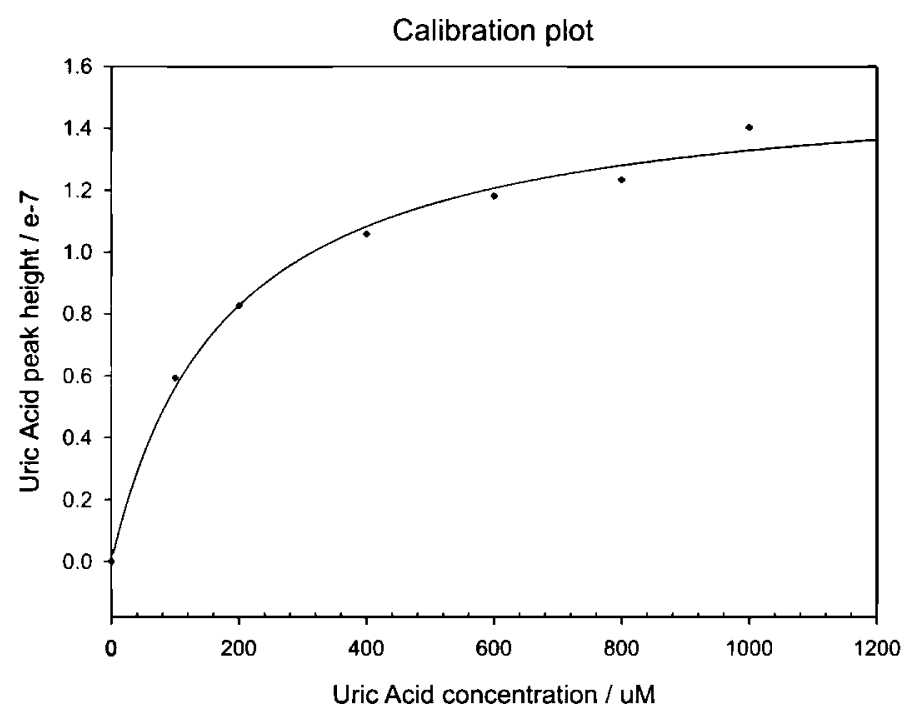

B)

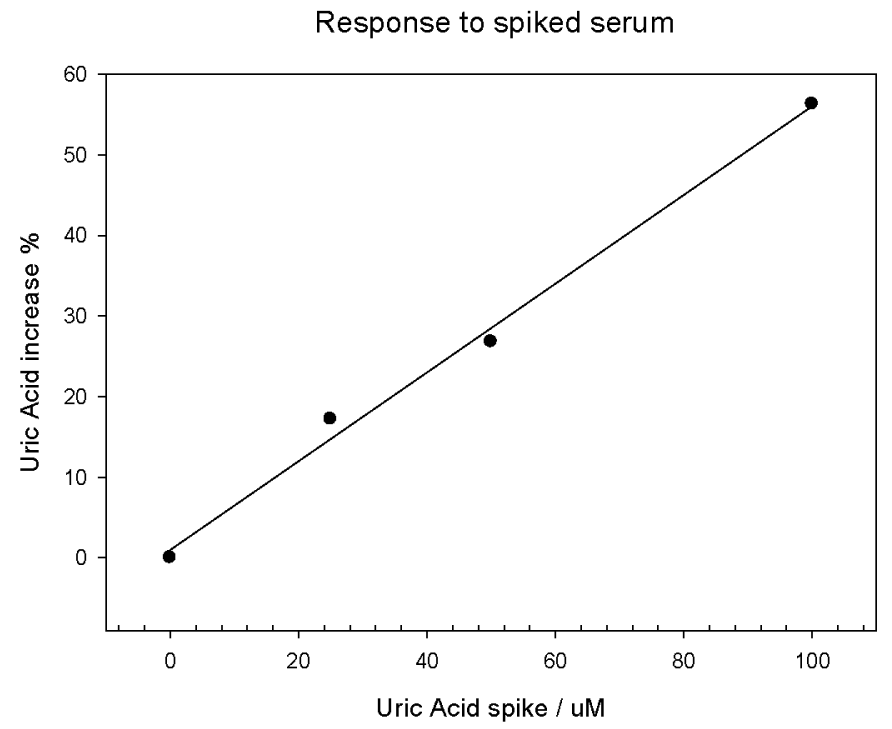

Figure 6. A) Calibration plot, with data, for the quantification of uric acid (in $\mathrm{pH} 7$ buffer) using an anodized carbon fibre sensor. B) Uric acid measurements in a serially spiked $(25,50$ and 100 $\mu \mathrm{M}$ urate) serum sample 